\title{
lleocolonic Lymphonodular Hyperplasia in Children Related to Etiologies Ranging from Food Hypersensitivity to Familial Mediterranean Fever
}

\author{
Murat Cakir Elif Sag $^{\mathrm{a}} \quad$ Ismail Saygin $^{\mathrm{b}}$ Fazil Orhan $^{\mathrm{c}}$ \\ a Department of Pediatric Gastroenterology Hepatology and Nutrition, Karadeniz Technical University, \\ Faculty of Medicine, Trabzon, Turkey; ${ }^{b}$ Department of Pathology, Karadeniz Technical University, Faculty of \\ Medicine, Trabzon, Turkey; ${ }^{c}$ Department of Pediatric Allergy and Immunology, Karadeniz Technical \\ University, Faculty of Medicine, Trabzon, Turkey
}

\section{Highlights of the Study}

- Lymphonodular hyperplasia (LNH) is a common finding in pediatric colonoscopies.

- It is mainly associated with food allergy, but irritable bowel syndrome, immunodeficiency, inflammatory bowel disease, familial Mediterranean fever, and the presence of $M E F V$ mutations may also be associated with ileocolonic LNH in children.

\section{Keywords}

Colon · Food hypersensitivity · Familial Mediterranean

fever - Lymphonodular hyperplasia

\begin{abstract}
Objective: We aimed to share our observations on the demographics, clinical characteristics, and outcomes of lymphonodular hyperplasia (LNH) in children. Subjects and Methods: The study included children on whom colonoscopy was performed between January 2015 and May 2018 ( $n=$ 361). Demographics, treatment modalities, and outcomes of the patients with LNH were recorded. Results: LNH was found in 66 patients (18.3\%; mean age $8.6 \pm 5.96$ years, $59.1 \%$ male). We found that the etiologic factors were food hypersensitivity ( $\mathrm{FH})$ in 25 (37.8\%), nonspecific colitis in 12 (18.2\%), irritable bowel syndrome in 10 (15.2\%), familial Mediterranean fever in 7 (10.6\%), primary immunodeficiency in 4
\end{abstract}

(6.1\%), and intestinal dysmotility, oxyuriasis, Crohn's disease, and giardiasis in $1(1.5 \%)$ patient. Additionally, in the genetic analysis of patients with idiopathic LNH $(n=4)$, we detected heterozygote MEFV mutations in all. Cow's milk and egg (25\%) were the most common allergens in patients with FH. Symptoms of all patients $(n=25)$ improved after an elimination diet. Conclusions: LNH is a common finding in pediatric colonoscopies with a variety of etiologies ranging from $\mathrm{FH}$ and familial Mediterranean fever to immunodeficiency.

(C) 2020 The Author(s)

Published by S. Karger AG, Basel

\section{Introduction}

Lymphoid aggregates are normally found throughout the gastrointestinal system; the number of these aggregates increases from the upper digestive system to the ter- karger@karger.com www.karger.com/mpp Karger $\stackrel{\text { ' }}{5}$

\section{(C) 2020 The Author(s)}

Published by S. Karger AG, Base

Karger

Open access

This is an Open Access article licensed under the Creative Commons Attribution-NonCommercial-4.0 International License (CC BY-NC) (http://www.karger.com/Services/OpenAccessLicense), applicable to the online version of the article only. Usage and distribution for commercial purposes requires written permission.
Murat Cakir

Karadeniz Technical University, Faculty of Medicine

Department of Pediatric Gastroenterology Hepatology and Nutrition

TR-31500 Ortahisar, Trabzon (Turkey)

muratcak@ hotmail.com 
Table 1. Demographic features and indications of colonoscopy in 361 patients

\begin{tabular}{|c|c|c|c|}
\hline \multirow[t]{2}{*}{$\begin{array}{l}\text { Age }^{\mathrm{a}} \text {, years (range) } \\
\text { Male gender } \\
\text { LNH }\end{array}$} & \multicolumn{2}{|c|}{$\begin{array}{l}9.7 \pm 5.4(1 \text { month to } 18 \text { years }) \\
190(52.8 \%) \\
66(18.3 \%) 95 \% \text { CI: } 14.3-22.9\end{array}$} & \multirow[b]{2}{*}{ Total $(n=361)$} \\
\hline & $\mathrm{LNH}(n=66)$ & Others $(n=295)$ & \\
\hline \multicolumn{4}{|l|}{ Indications of colonoscopy } \\
\hline Rectal bleeding & $23(34.8 \%)$ & $117(39.6 \%)$ & $140(38.8 \%)$ \\
\hline Chronic abdominal pain & $10(15.2 \%)$ & $76(25.7 \%)$ & $86(23.8 \%)$ \\
\hline Chronic diarrhea & $14(21.2 \%)$ & $40(13.5 \%)$ & $54(15 \%)$ \\
\hline Persistent anemia & $4(6.1 \%)$ & $26(8.8 \%)$ & $30(8.3 \%)$ \\
\hline FMF patients ${ }^{\mathrm{b}}$ & $5(7.6 \%)^{c}$ & $6(2 \%)^{c}$ & $11(3 \%)$ \\
\hline Growth retardation & $4(6.1 \%)$ & $6(2 \%)$ & $10(2.8 \%)$ \\
\hline Bloody diarrhea & 0 & $8(2.7 \%)$ & $8(2.2 \%)$ \\
\hline Chronic anal fistula & 0 & $6(2 \%)$ & $6(1.7 \%)$ \\
\hline Chronic constipation & $5(7.6 \%)^{c}$ & $0^{c}$ & $5(1.4 \%)$ \\
\hline Polyp screening & 0 & $7(2.3 \%)$ & $7(1.9 \%)$ \\
\hline Weight loss & 0 & $1(0.3 \%)$ & $2(0.6 \%)$ \\
\hline Malignancy screening & 0 & $1(0.3 \%)$ & $1(0.3 \%)$ \\
\hline IBS-related symptoms & $1(1.5 \%)$ & 0 & $1(0.3 \%)$ \\
\hline
\end{tabular}

Values express $n$, unless otherwise indicated. FMF, familial Mediterranean fever; IBS, irritable bowel syndrome; LNH, lymphoid nodular hyperplasia.

${ }^{\mathrm{a}}$ Mean $\pm \mathrm{SD}$; ${ }^{\mathrm{b}}$ these patients were evaluated for chronic gastrointestinal system symptoms while receiving colchicine treatment; ${ }^{c} p<0.05$.

minal ileum and appendix, and the colon also contains a considerable number of lymphoid aggregates. They are evident as large numbers $(>10)$ of lymphoid nodules with a diameter of $2-10 \mathrm{~mm}$ and a flat and yellow-white appearance during colonoscopy that defines them as lymphonodular hyperplasia (LNH). Histopathologic examination reveals hyperplastic lymphoid follicles and mitotically active germinal centers with lymphocytic mantles $[1,2]$.

$\mathrm{LNH}$ is a common finding during pediatric colonoscopy, but its clinical significance and etiologic factors are unclear. Previous studies have defined the association of LNH with food allergy, infections, and immunodeficiency syndromes in adults [3-6]. Additionally, it has been shown to have an association with connective tissue diseases, inflammatory bowel disease (IBD), juvenile idiopathic arthritis, lymphoma, and refractory constipation [3]. Interestingly, an association was shown between LNH and autism due to chronic constipation [7]. It can also be seen in normal individuals without any symptoms or may be related to nonspecific chronic abdominal symptoms $[3,8,9]$.

Here, we describe the demographic and clinical characteristics and outcomes of LNH in pediatric patients.

\section{Subjects and Methods}

This is a retrospective study for which data were obtained from hospital files. It included all patients $(<18$ years of age) who had undergone full colonoscopy including ileal intubation between January 2015 and May 2018 in a pediatric gastroenterology unit of a tertiary hospital. The demographic characteristics, symptoms, laboratory, endoscopic and histopathologic findings, treatment modalities, and outcomes of the patients with LNH were recorded. All colonoscopic procedures were performed on a Pentax EPK-100 device (HOYA Corp., Tokyo, Japan) by M.C. and E.S.

Diagnosis of LNH was based on the presence of an endoscopic appearance and/or histopathologic findings of LNH. LNH was defined as: (i) $>10$ lymphoid nodules with a diameter $>2 \mathrm{~mm}$ on colonoscopic examination, and (ii) hyperplastic lymphoid follicles and mitotically active germinal centers with lymphocytic mantles on histopathologic examination $[5,10]$. All colonoscopic procedures were performed on a Pentax device (HOYA Corp.). Biopsies of abnormal lesions were obtained in all sections of the colon (in the rectum and sigmoid, descending, transverse, and ascending colon) and terminal ileum even when these appeared normal.

Depending on the individual clinical and laboratory data, a diagnostic workup was done including complete blood count, complete biochemical tests, stool microscopy, immunologic tests, and possible infectious etiologies (stool culture for bacteria, viruses, and parasites; serum PCR for EBV, CMV, and HIV). Skin prick tests and/or food-specific immunoglobulin E (IgE) tests were also performed. Patients with positive results, except for patients $<1$ year old, were put on an elimination diet elimination 
Fig. 1. Lymphonodular hyperplasia of the terminal ileum (a) and colon (b).
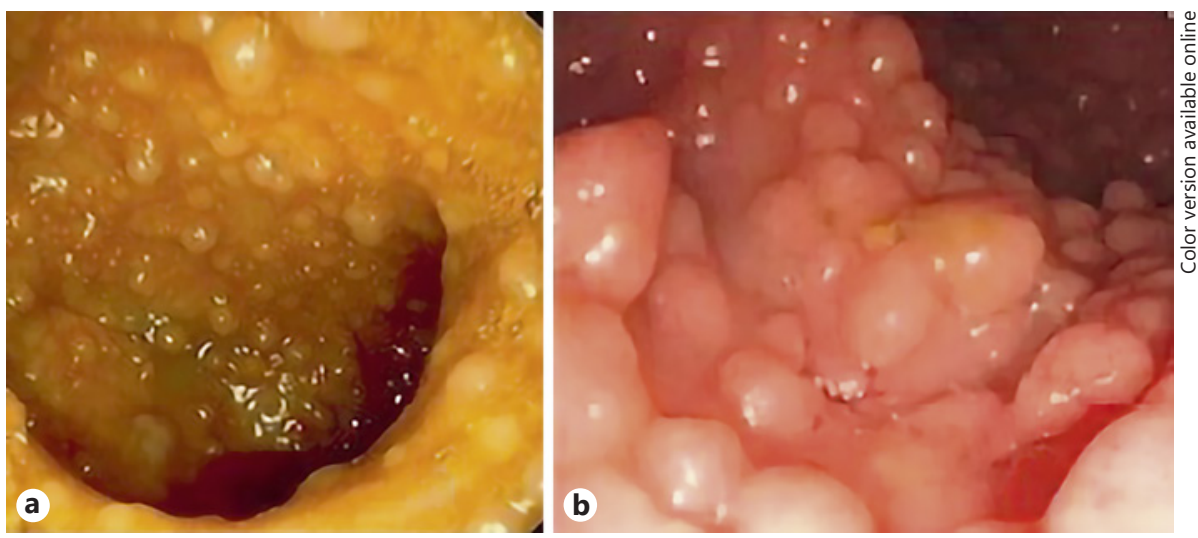

according to the test findings. Younger patients underwent a therapeutic 2 -food diet challenge (cow's milk and egg) with formula or breast milk regardless of the test findings. A positive clinical response was accepted as food hypersensitivity (FH). According to previous studies, $\mathrm{LNH}$ is a common manifestation of familial Mediterranean fever (FMF) [11]. Therefore, we evaluated the patients with idiopathic LNH for FMF via clinical and/or genetic analysis. A final diagnosis of FMF was made, according to the Tel Hashomer criteria [12], by a pediatric rheumatologist. The diagnosis of irritable bowel syndrome (IBS) was made by clinical parameters as defined previously [13]. Skin prick test and/or foodspecific IgE were negative in patients with IBS. Nonspecific colitis was defined as an increase in chronic inflammatory cells without architectural abnormalities, the presence of multiple basal lymphoid aggregates or basal inflammatory cells immediately above the muscularis mucosae, and no reactive changes on the surface epithelium or in the crypts [14]. The diagnosis of other conditions such as IBD (including Crohn's disease, ulcerative colitis, and indeterminate colitis), infectious colitis, and microscopic colitis were excluded before a diagnosis of nonspecific colitis was made. Skin prick test and/or food-specific IgE were negative in these patients.

All calculations in our study were performed using the Statistical Package for Social Sciences v23 (IBM Corp., Armonk, NY, USA), and the continuous variables were expressed as mean \pm standard deviation and categorical variables as $n(\%)$.

\section{Results}

A total of 401 colonoscopies were performed on 361 patients during the study period. LNH was found in 66 patients (18.3\%, 95\% CI 14.3-22.9) (Fig. 1, 2). Demographic features and indications for the colonoscopy are shown in Table 1. LNH was detected in the terminal ileum in 40 patients $(60.6 \%)$, in the colon in $19(28.8 \%)$, and in both the ileum and colon in 7 (10.6\%). Additional to $\mathrm{LNH}$, ileal aphthous ulcers in 2 patients (3\%) and colonic ulcers in 2 patients (3\%) were detected.

Fig. 2. Terminal ileum biopsy: hyperplasic lymphoid follicles, suggesting nodular lymphoid hyperplasia. HE. $\times 1,000$.

After all examinations, we found that the etiologic factors in LNH were FH in 25 (37.8\%), nonspecific colitis in 12 patients (18.2\%), IBS in 10 (15.2\%, constipation-dominant in 5 and diarrhea-dominant in 5), FMF in 7 (10.6\%), and primary immunodeficiency in 4 (6.1\%). Intestinal dysmotility, oxyuriasis, and giardiasis were detected in 1 patient (1.5\%). Five of the seven patients with FMF were receiving colchicine treatment and had chronic gastrointestinal system symptoms (FMF-related LNH). After the colonoscopy, 2 patients with LNH were diagnosed as having FMF by clinical and/or genetic analysis (primary FMF-related LNH). Additionally, in the genetic analysis of patients with idiopathic LNH $(n=4,6.1 \%)$, we detected heterozygote $M E F V$ mutations ( 2 of G304P and 1 of 
Table 2. Demographic features and etiology of patients with LNH $(n=66)$

\begin{tabular}{|c|c|}
\hline $\begin{array}{l}\text { Age }^{\mathrm{a}} \text {, years (range) } \\
\text { Male gender }\end{array}$ & $\begin{array}{l}8.6 \pm 5.96 \text { ( } 2 \text { months to } 18 \text { years) } \\
39(59.1)\end{array}$ \\
\hline \multicolumn{2}{|l|}{ Etiology } \\
\hline Food hypersensitivity & $25(37.8)$ \\
\hline Nonspecific colitis & $12(18.2)$ \\
\hline IBS (constipation/diarrhea dominant) & $10(15.2)(5 / 5)$ \\
\hline FMF (FMF-related LNH/primary FMF-related LNH) & $7(10.6)(5 / 2)$ \\
\hline CVID & $4(6.1)$ \\
\hline Dysmotility (operated HD) & $1(1.5)$ \\
\hline Giardiasis & $1(1.5)$ \\
\hline Oxyuriasis & $1(1.5)$ \\
\hline Crohn's disease & $1(1.5)$ \\
\hline Idiopathic LNH (heterozygous $M E F V$ mutation) & $4(6.1)$ \\
\hline
\end{tabular}

Values express $n(\%)$, unless otherwise indicated. CVID, common variable immune deficiency; FMF, familial Mediterranean fever; IBS, irritable bowel syndrome; HD, Hirschsprung disease.

${ }^{\text {a }}$ Mean \pm SD.

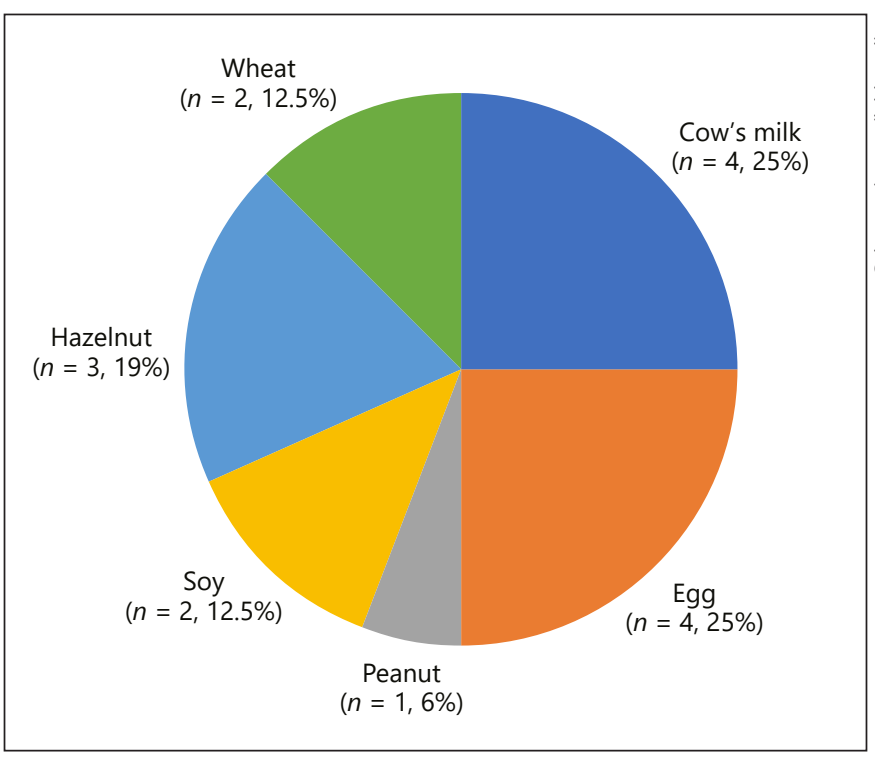

Fig. 3. Distribution of food allergens.

E148Q and L110P) in 4 patients. But they did not have any clinical findings compatible with FMF during the follow-up. The demographic features and etiology of patients with LNH are shown in Table 2.

There were 25 patients with suspected $\mathrm{FH}$. Twelve of these were treated with an elimination diet according to positive skin prick test and/or food-specific IgE findings. In total, 16 allergens were positive, and 4 patients had multiple food allergies. Cow's milk and egg (25\%) were the most common allergens (Fig. 3). Additionally, aeroal- lergens were positive in 5 patients. Skin prick test and/or food-specific IgE were negative in 13 patients. All of these patients were younger than 1 year and had clinical findings compatible with allergic proctocolitis. They underwent an empiric 2-food elimination diet (cow's milk and egg). Symptoms of all patients $(n=25)$ including rectal bleeding in 19 , chronic abdominal pain in 3 , chronic diarrhea in 2 , and chronic constipation in 1 improved with the elimination diet.

The patients with primary immunodeficiency $(n=4)$ were initially admitted to our clinic because of their chronic gastrointestinal symptoms (chronic diarrhea in 2 and bloody diarrhea in 2). We found decreased immunoglobulin levels in all 4 of them. They were diagnosed as having common variable immunodeficiency (CVID) and were treated with periodic intravenous immunoglobulin therapy. Patients with IBS and dysmotility $(n=11)$ were treated with a prokinetic/laxative according to the dominant type. Additionally, patients with nonspecific colitis $(n=12)$ were treated with mesalamine therapy (an oral daily dose of $50 \mathrm{mg} / \mathrm{kg}$ ). The patients with primary FMFrelated LNH $(n=2)$ were treated with colchicine (1-2 mg/ $\mathrm{kg})$. No additional treatment was prescribed for patients with FMF-related LNH $(n=5)$.

The final diagnosis of patients with ileal aphthous ulcers were Crohn's disease and food allergy; patients with colonic ulcers had FMF-related LNH and CVID. Patients with idiopathic $\mathrm{LNH}$ and heterozygous $M E F V$ mutation were monitored in outpatient clinics without any treatment. 


\section{Discussion}

In this study, we present the demographic and clinical findings, etiologies, and outcomes of children with LNH. We found that: (i) it is a common finding in pediatric colonoscopies (18.3\%), (ii) the majority of LNH cases are associated with FH (37.8\%), (iii) in addition to FH and IBS (15.2\%), immunodeficiency (6.1\%), IBD (1.5\%), FMF (7.6\%), and carrying $M E F V$ mutations (6.1\%) may be associated with ileocolonic $\mathrm{LNH}$, and (iv) most of the patients improved after the underlying condition was treated.

Luminal commensals and pathogenic flora induce the production of specific IgA antibodies within Peyer's patches and lymphoid follicles. In this response, lymphoid cells accumulate and induce the evolution of $\mathrm{M}$ cells in the surface epithelium. Secreted IgA is induced by the stimulation of luminal factors such as chronic inflammation, infections, or protein antigens. An imbalance or dysfunction in the secretion of luminal IgA has been suggested to cause LNH [15]. LNH in association with immunodeficiency might be explained as a compensatory mechanism. Similarly, an increase in the number of lymphoid follicles in the colon of patients with colitis (nonspecific, IBD, or infectious) may represent similar attempts to control the inflammation by increased production of IgA. Mechanisms linking food allergy to LNH are not clear. An association of a history of non-IgE-mediated food allergy in infancy and an increase in antiallergen IgA levels has been shown. Additionally, deficient Thelper 1 function has been reported in the peripheral blood of patients with LNH (similar to in patients with delayed-type food allergy), such as increased interleukin (IL)-6 messenger RNA (mRNA) and interferon (IFN)- $\gamma$ mRNA in the colonic mucosa and increased IFN- $\gamma$, IL-4, and IL-10 secretion in the periphery [16]. Diet-related changes in the microbiome (in IBD) and longer exposure to mucosal content (in dysmotility syndromes) may also induce unbalanced IgA secretion [15].

LNH is a common finding during pediatric colonoscopies. Gurkan et al. [11] reported that LNH was found $12.6 \%$ in children who underwent colonoscopy. Lucarelli et al. [17] reported an increased ratio in Italian children, i.e., $36 \%$. In previous studies, the frequency of LNH varied between 10 and $30 \%$, with variation depending on the terminal ileum intubation, colonoscopy indications, and study design (e.g., including only isolated LNH) [10].

Although LNH is considered a physiological condition, the association of $\mathrm{LNH}$ and $\mathrm{FH}$ has also been extensively studied. The association is more evident in colonic LNH when compared to LNH localized to the terminal ileum.
Mansueto et al. [3] reviewed the link between LNH and $\mathrm{FH}$, and found that approximately $66 \%$ of patients with LNH had FH and $49 \%$ of patients with FH had LNH. The most common symptoms in patients with LNH associated with $\mathrm{FH}$ are chronic abdominal pain, chronic diarrhea, hematochezia, and a failure to thrive. Increased levels of lamina propria $\gamma \delta+$ T cells can be detected in histopathologic examinations of the patients [18]. Generally, patients are of school age or preschool age, and have multiple food allergies and elevated serum anti- $\beta$-lactoglobulin $\operatorname{IgG}[3,19]$.

Lucarelli et al. [17] reported a weak association between LNH and FH. They divided the isolated LNH patients into 3 groups randomly: an elimination diet group (cow's milk, eggs, and foods positive on skin prick tests), a mesalamine group, and a symptomatic treatment group. At the end of the 8-week treatment, there was no significant difference in response to treatment among the groups. No association was found between the treatment response and the clinical, endoscopic, and allergic features of the patients. In our study, 37.8\% of the patients with LNH had FH. The lack of a double-blind placebo control challenge test or control colonoscopic examinations made the diagnosis of food allergy in our patients questionable, but the symptoms of all patients improved after the elimination diet. We suggest that $\mathrm{LNH}$ is a manifestation of FH. It is also a common finding in allergic proctocolitis and nonIgE-mediated food allergy in infants. Our study did not indicate an association between LNH and non-IgE mediated food allergy in older children.

A study performed at our center revealed that $\mathrm{LNH}$ is the most common colonoscopic finding $(27.8 \%)$ in patients with FMF [20]. Another study reported that FMF is responsible for $\mathrm{LNH}$ etiology in $15 \%$ of patients [11]. All the patients had compatible clinical findings and the diagnosis was confirmed by genotype analysis in these patients. Additionally, the authors showed that patients with FMF-related LNH had higher sedimentation rates and C-reactive protein levels, and more perianal lesions compared the patients without FMF. All of them were treated with colchicine treatment [11]. In our study, $7 \mathrm{pa}$ tients (10.6\%) had FMF and 5 of these received colchicine treatment. Two patients were diagnosed during the follow-up. Interestingly, 4 patients (6.1\%) with LNH had a heterozygote MEFV genotype. We suggest that low-grade intestinal inflammation may persist in patients with FMF despite treatment and, in addition, low-grade intestinal inflammation may present in individuals with the heterozygote genotype. It has been shown that patients with FMF on colchicine treatment have increased levels of fecal calprotectin compared to healthy controls, suggesting 
low-grade intestinal inflammation [21]. Increased inflammatory symptoms have been found in individuals with a heterozygote MEFV genotype [22].

$\mathrm{LNH}$ of the colon and bulbus is a common finding with primary immunodeficiency, especially CVID and selective IgA deficiency [23]. In adults, LNH was found in $20 \%$ of patients with combined immunodeficiencies [11,24]. But data on pediatric patients are scarce. Gurkan et al. [11] reported that the frequency of immunodeficiency associated with isolated LNH is $2.5 \%$. In our study, we found it was $6.1 \%$. Opportunistic chronic parasitic or viral infections may lead to the gastrointestinal symptoms and development of $\mathrm{LNH}$ in patients with immunodeficiency.

IBS is another cause of LNH in children. Approximately $15 \%$ of our patients with LNH were diagnosed with IBS based on the clinical diagnostic criteria. In adults, approximately $1 / 3$ patients with IBS-type symptoms or suspected IBD present with diffuse colonic LNH. IBD and IBS are suggested to be different presentation of the same pathology. "Minimal lesion colitis," also called colonic LNH is a common feature of IBS, IBD, Ni-allergy syndrome and other conditions such as hypersensitivity reactions, immunodeficiency, and chronic gastrointestinal infections. The factors and pathogenesis involved in the development of different clinical features from the same pathology are still under evaluation [25].

The association between LNH and chronic parasitic infections such as giardiasis were reported in previous studies. The presence of both giardiasis and hypogammaglobulinemia may cause the development of LNH. In our study, giardiasis and oxyuriasis were shown in a minority of patients but none of them had hypogammaglobulinemia.

\section{Conclusion}

We found that LNH may be related to many diseases such as FH, FMF, IBS, and immunodeficiency in children. Elimination of the triggering foods improved symptoms in patients with FH. The association of $\mathrm{LNH}$ and FMF is interesting, and FMF should be included in the list of causative factors of LNH especially in the presence of other clinical findings. Sometimes LNH may be a response to functional problems such as chronic constipation and diarrhea.

\section{Statement of Ethics}

Patients in the study have given their written informed consent and that the study protocol was approved by the institute's committee on human research (study No. 2018/285).

\section{Disclosure Statement}

The authors have no conflicts of interest to declare.

\section{Funding Sources}

No funding was received for this study.

\section{Author Contributions}

M.C. and E.S. designed and wrote the manuscript; I.S. and F.O. did the clinical examinations.

\section{References}

1 Kuper CF. Histopathology of mucosa-associated lymphoid tissue. Toxicol Pathol. 2006; 34(5):609-15.

2 Kunisawa J, Fukuyama S, Kiyono H. Mucosaassociated lymphoid tissues in the aerodigestive tract: their shared and divergent traits and their importance to the orchestration of the mucosal immune system. Curr Mol Med. 2005 Sep;5(6):557-72.

3 Mansueto P, Iacono G, Seidita A, D'Alcamo A, Sprini D, Carroccio A. Review article: intestinal lymphoid nodular hyperplasia in children-the relationship to food hypersensitivity. Aliment Pharmacol Ther. 2012 May;35(9):1000-9.

4 Krauss E, Konturek P, Maiss J, Kressel J, Schulz U, Hahn EG, et al. Clinical significance of lymphoid hyperplasia of the lower gastrointestinal tract. Endoscopy. 2010 Apr;42(4): $334-7$.
5 Albuquerque A. Nodular lymphoid hyperplasia in the gastrointestinal tract in adult patients: A review. World J Gastrointest Endosc. 2014 Nov;6(11):534-40.

6 Carroccio A, Iacono G, Di Prima L, Ravelli A, Pirrone $G$, Cefalù $A B$, et al. Food hypersensitivity as a cause of rectal bleeding in adults. Clin Gastroenterol Hepatol. 2009 Jan;7(1): $120-2$.

7 MacDonald TT, Domizio P. Autistic enterocolitis; is it a histopathological entity? Histopathology. 2007 Feb;50(3):371-9.

8 Kojima M, Nakamura N, Itoh H, Motoori T, Hoshi K, Enomoto Y, et al. Histological variety of localized lymphoid hyperplasia of the large intestine: histopathological, immunohistochemical and genotypic findings of 16 cases. J Clin Exp Hematop. 2009 May;49(1): $15-21$.
9 Monsanto P, Lérias C, Almeida N, Lopes S, Cabral JE, Figueiredo P, et al. Intestinal nodular lymphoid hyperplasia and extraintestinal lymphoma-a rare association. Acta Gastroenterol Belg. 2012 Jun;75(2):260-2.

10 Iacono G, Ravelli A, Di Prima L, Scalici C, Bolognini S, Chiappa S, et al. Colonic lymphoid nodular hyperplasia in children: relationship to food hypersensitivity. Clin Gastroenterol Hepatol. 2007 Mar;5(3):361-6.

11 Gurkan OE, Yilmaz G, Aksu AU, Demirtas Z, Akyol G, Dalgic B. Colonic lymphoid nodular hyperplasia in childhood: causes of familial Mediterranean fever need extra attention. J Pediatr Gastroenterol Nutr. 2013 Dec;57(6): 817-21.

12 Pras M. Familial Mediterranean fever: from the clinical syndrome to the cloning of the pyrin gene. Scand J Rheumatol. 1998;27(2):92-7. 
13 Devanarayana NM, Rajindrajith S. Irritable bowel syndrome in children: current knowledge, challenges and opportunities. World J Gastroenterol. 2018 Jun;24(21):2211-35.

14 Ojuawo A, St Louis D, Lindley KJ, Milla PJ. Non-infective colitis in infancy: evidence in favour of minor immunodeficiency in its pathogenesis. Arch Dis Child. 1997 Apr; 76(4):345-8.

15 Knoop KA, Newberry RD. Isolated Lymphoid Follicles Are Dynamic Reservoirs for the Induction of Intestinal IgA. Front Immunol. 2012 May;3:84.

16 Paajanen L, Vaarala O, Karttunen R, Tuure T, Korpela R, Kokkonen J. Increased IFN-gamma secretion from duodenal biopsy samples in delayed-type cow's milk allergy. Pediatr Allergy Immunol. 2005 Aug;16(5):439-44.

17 Lucarelli S, Lastrucci G, Di Nardo G, D’Alfonso Y, Aloi M, Oliva S, et al. Intestinal lymphoid nodular hyperplasia in children: the relationship to food allergy. Pediatr Allergy Immunol. $2015 \mathrm{Feb}$;26(1):18-24.
18 Kokkonen J, Holm K, Karttunen TJ, Mäki M; J. Kokkonen, K. Holm, T. J. Karttun. Children with untreated food allergy express a relative increment in the density of duodenal gammadelta+ T cells. Scand J Gastroenterol. 2000 Nov;35(11):1137-42.

19 Kokkonen J, Tikkanen S, Karttunen TJ, Savilahti E. A similar high level of immunoglobulin A and immunoglobulin G class milk antibodies and increment of local lymphoid tissue on the duodenal mucosa in subjects with cow's milk allergy and recurrent abdominal pains. Pediatr Allergy Immunol. 2002 Apr; 13(2):129-36

20 Sağ E, Demir F, Saygın İ, Kalyoncu M, Çakır $\mathrm{M}$. Endoscopic Findings of Children with Familial Mediterranean Fever. Pediatr Gastroenterol Hepatol Nutr. 2018 Oct;21(4):271-7.

21 Gucenmez OA, Kume T, Makay B, Babayigit O, Arslan N, Unsal E. Role of fecal calprotectin in the assessment of intestinal inflammation in children with familial Mediterranean fever. Int J Rheum Dis. 2018 Oct;21(10): 1844-8.
22 Kalyoncu M, Acar BC, Cakar N, Bakkaloglu A, Ozturk S, Dereli E, et al. Are carriers for MEFV mutations "healthy"? Clin Exp Rheumatol. 2006 Sep-Oct;24(5 Suppl 42):S120-2.

23 Agarwal S, Mayer L. Gastrointestinal manifestations in primary immune disorders. Inflamm Bowel Dis. 2010 Apr;16(4):703-11.

24 Sauerbrei E, Castelli M. Hypogammaglobulinemia and nodular lymphoid hyperplasia of the gut. J Can Assoc Radiol. 1979 Mar;30(1): 62-3.

25 Piscaglia AC, Laterza L, Cesario V, Gerardi V, Landi R, Lopetuso LR, et al. Nodular lymphoid hyperplasia: A marker of low-grade inflammation in irritable bowel syndrome? World J Gastroenterol. 2016 Dec;22(46): 10198-209.

26 Webster AD. Giardiasis and immunodeficiency diseases. Trans R Soc Trop Med Hyg. 1980;74(4):440-3. 